\title{
Validez de constructo y confiabilidad de la versión en español del Spirituality Index of Well-Being (SIWB) en población colombiana con enfermedad pulmonar crónica
}

\author{
Construct validity and reliability of the Spanish \\ version of Spirituality Index of Well-Being (SIWB) in \\ Colombian population with chronic lung disease
}

Stefano Vinaccia Alpi ${ }^{1}$, Fernando Riveros Munévar ${ }^{2}$, Japcy Margarita Quiceno ${ }^{3}$

Forma de citar: Vinaccia Alpi S, Riveros Munévar F, Quiceno JM. Validez de constructo y confiabilidad de la versión en español del Spirituality Index of Well-Being (SIWB) en población colombiana con enfermedad pulmonar crónica. Rev Univ Ind Santander Salud. 2016; 48(3): 321-330. DOI: http://dx.doi.org/10.18273/revsal.v48n3-2016006 @c) (1) €

\section{RESUMEN}

Introducción: La enfermedad pulmonar obstructiva crónica (EPOC) es un problema de salud pública que está aumentando en Colombia, con altos costos sociales y económicos, sin contar con instrumentos para medir sus condiciones directamente. Objetivo: Estudiar la validez de constructo y la confiabilidad de la versión en español del cuestionario Spirituality Index of Well-Being (SIWB) en una muestra colombiana de pacientes con diagnóstico de enfermedad crónica pulmonar. Metodología: Estudio de corte transversal, de alcance descriptivo y de tipo instrumental, donde se aplicó el cuestionario a 71 pacientes adultos con diagnóstico de enfermedad pulmonar obstructiva crónica de la ciudad de Bogotá. Se realizaron análisis factoriales por método de extracción de componentes principales, con rotación Varimax, así como análisis de consistencia por alfa de Cronbach para la escala general y cada factor obtenido, correlaciones inter-elemento y estadísticos total-elemento. Resultados: El análisis factorial denota dos factores: autoeficacia $(38,884 \%$ de varianza explicada) y esquemas vitales $(37,557 \%$ de varianza explicada), factores que explican el 76,440\% de la varianza total acumulada y que concuerdan con la propuesta teórica de las escalas, así como un alfa de Cronbach general de 943 y correlaciones positivas entre todos los reactivos. Conclusiones: La escala muestra una alta confiabilidad, apropiada consistencia interna entre los reactivos, concordancia entre elementos-total corregida, y concordancia en la estructura factorial con la versión original, lo que sugiere una adecuada validez de constructo de la prueba, con apropiada confiabilidad y consistencia en población colombiana de enfermos crónicos.

Palabras clave: Validez de las pruebas, reproducibilidad de resultados, espiritualidad, enfermedades pulmonares, psicometría.

1. Fundación Universitaria Sanitas. Bogotá, Colombia.

2. Universidad de San Buenaventura. Bogotá, Colombia

3. Universidad de Medellín. Bogotá, Colombia

Correspondencia: Fernando Riveros Munévar. Dirección: Carrera 8H 172 -20 Bogotá, Colombia. Correo electrónico: efriveros45@hotmail. com. Teléfono 3007709516. 


\section{ABSTRACT}

Introduction: Chronic obstructive pulmonary disease (COPD) is a public health problem that is increasing in Colombia, with high social and economic costs, without instruments to measure their conditions directly. Objective: To study the construct validity and reliability of the Spanish version of the questionnaire Spirituality Index of Well-Being (SIWB) in a Colombian sample of patients diagnosed with chronic lung disease. Methodology: Crosssectional study, descriptive scope and instrument type, where applied the questionnaire to 71 adult patients diagnosed with chronic obstructive pulmonary disease in Bogota. Factor analyzes were performed by extraction method of principal components with Varimax rotation and analysis of consistency by Cronbach's alpha for the overall scale and each factor obtained inter-item correlations and statistical full-element. Results: Factor analysis indicates two factors: self-efficacy (38.884\% of explained variance) and life patterns (37.557\% of explained variance), factors explaining $76,440 \%$ of the total cumulative variance and consistent with the theoretical proposal of the scales, as well as a general Cronbach, 943 and positive correlations between all reagents. Conclusions: The scale shows high reliability, appropriate internal consistency between the reactants, concordance between elements-all corrected, and agreement on the factorial structure with the original version, suggesting adequate construct validity of the test, with appropriate reliability and consistency Colombian population of chronically ill.

Keywords: Validity of Tests, reproducibility of results, spirituality, lung diseases, psychometrics.

\section{INTRODUCCIÓN}

La Enfermedad pulmonar obstructiva crónica (EPOC) es un término utilizado para describir dos enfermedades pulmonares relacionadas: la bronquitis crónica y el enfisema pulmonar. La bronquitis crónica se caracteriza por la inflamación prolongada del interior de los bronquios (los conductos de las vías respiratorias) y el enfisema es el ensanchamiento y destrucción irreversible de los alvéolos pulmonares (sacos aéreos). Es posible que algunas personas con EPOC manifiesten ambas enfermedades ${ }^{1,2}$.

La enfermedad pulmonar obstructiva crónica (EPOC) es un problema de salud pública de enormes proporciones que está aumentando en todo el mundo por su morbimortalidad y costos sociales y económicos. En los últimos 30 años la mortalidad mundial por EPOC ha aumentado 163\%. La Organización Mundial de la Salud $d^{2,3,4,5}$ calcula que para el año 2020 la EPOC pasará de ser la causa número 12 de enfermedad en el mundo a la quinta causa de años de vida perdidos, ajustados por invalidez, en el mismo periodo de tiempo anterior la mortalidad mundial por EPOC ha aumentado 163\%. La Organización Mundial de la Salud ${ }^{4}$ calcula que para el año 2020 la EPOC pasará de ser la causa número 12 de enfermedad en el mundo a la quinta causa de años de vida perdidos, ajustados por invalidez $z^{6,7,8,9}$.
Frente al afrontamiento de enfermedades crónicas, como es el caso del EPOC, se ha hallado que el papel de la espiritualidad como estrategia de afrontamiento en estados avanzados y críticos de la enfermedad favorece la calidad de vida y la regulación de la afectividad negativa ${ }^{10}$. Por otro lado, Pargament ${ }^{10}$ basado en los planteamientos de Lazarus y Folkman sobre afrontamiento al estrés comienza a desarrollar desde finales de los años ochenta y que puntualiza en 1997 su modelo de "Religious coping", "afrontamiento religioso" en español, el cual describió como aquel "tipo de afrontamiento donde se utilizan creencias y comportamientos religiosos para prevenir y/o aliviar las consecuencias negativas de sucesos de vida estresantes, tanto como para facilitar la resolución de problemas". El modelo de Pargament ${ }^{10}$ hace referencia a tres estilos de afrontamiento religioso como: 1. estilo autodirigido, en el cual las personas confían en sí mismas más que en Dios para resolver sus problemas; 2. estilo elusivo o evitativo, en la que la responsabilidad es dejada en manos de la divinidad, y 3. estilo colaborativo, en la que se establece una dinámica compartida entre el hombre y Dios en el proceso de afrontamiento. Estos tres procesos están también asociados al nivel de competencia, fundamentalmente al sentido de control personal, la habilidad para la solución de problemas, y la autoestima. Así, el estilo 
autodirigido estaría asociado a un bajo sentido de competencia personal y alta autoestima, con tendencia a la autonomía. El estilo evitativo estaría asociado a un bajo sentido de competencia personal, baja autoestima y habilidad de solución de problemas, así como mayor intolerancia a las diferencias interpersonales; enfatizando la autoridad externa, y constituyéndose en un estilo pasivo. Finalmente, el estilo colaborativo estaría asociado a un elevado sentido de control personal y autoestima, y bajo sentido de control por las circunstancias o el azar ${ }^{11,12}$.

Las investigaciones basadas en la evidencia plantean además que aunque las personas no sean sumamente religiosas en su diario vivir, pueden serlo en momentos de enfermedad, debido a la experiencia de pérdida de control personal frente a la situación, que los lleva a la búsqueda de un poder más alto o un Dios para encontrar los propósitos de la vida y enfrentar situaciones estresantes $^{13}$.

Esto ha llevado en los últimos años al desarrollo de modelos que consideran el bienestar espiritual como un componente esencial de la calidad de vida relacionada con la salud pero distinto de los dominios tradicionales físicos, sociales y mentales. Este nuevo modelo BioPsico-Social-Espiritual puede ser útil en contextos clínicos para evaluar la el bienestar espiritual de los pacientes que puede ayudar a dar sentido a sus vidas y sentir esperanza y paz aun en medio de una enfermedad grave $^{14}$

En ese sentido, Strada, et al. ${ }^{15}$ desarrollaron un estudio longitudinal con 103 pacientes de EPOC que vinieron entrevistados cada tres meses durante un periodo de 30 meses. Los resultados encontraron que el bienestar espiritual tenía una importante relación positiva con la calidad de vida de estos pacientes.

$\mathrm{Al}$ respecto Quiceno, et al. ${ }^{16}$ realizaron una revisión donde concluyen que son muchas las investigaciones que muestran a la religión y la espiritualidad como variables moderadoras y amortiguadoras ante eventos traumáticos de la vida, como puede ser una enfermedad, afirmación que Daaleman, et a $1^{17}$ comparten, planteando además que la espiritualidad no requiere la inclusión de una religión o un Dios, lo cual favorece la evaluación de este constructo, aun en pacientes escépticos o declarados como ateos.
En esta revisión se encontró que no existían instrumentos psicométricos válidos y confiables para evaluar bienestar espiritual en poblaciones de adultos mayores en Colombia cuando desde un punto de vista social y cultural, la espiritualidad y la religión son especialmente relevantes en la vida de amplios segmentos de población colombiana especialmente en muestras de pacientes con diagnóstico de enfermedad crónica. Esto llevó a desarrollar una investigación, analizar las características psicométricas con un instrumento como el SIWB que mide el bienestar psicológico espiritual en pacientes con enfermedades físicas y puede emplearse en estudios que evalúen calidad de vida. El objetivo del siguiente trabajo fue por lo tanto la de evaluar psicométricamente el índice de bienestar psicológico ${ }^{17}$ en una muestra de Pacientes de EPOC de la ciudad de Bogotá.

\section{METODOLOGÍA}

\section{Diseño}

El presente es un estudio de corte transversal, de alcance descriptivo y de tipo instrumental ${ }^{18}$.

\section{Participantes}

Participaron en este estudio 71 pacientes adultos de manera libre y voluntaria, con diagnóstico de enfermedad pulmonar obstructiva crónica EPOC, quienes fueron escogidos mediante un muestreo no aleatorio por sujetos disponibles.

Dichos participantes estaban siendo atendidos en una clínica privada de la ciudad de Bogotá, los cuales se contactaron vía telefónica directamente desde la clínica, en donde se les comentó del estudio y se les invitó a participar del mismo, teniendo en cuenta dichos pacientes no tuvieran limitaciones cognitivas, enfermedades concomitantes o trastornos psiquiátricos que pudieran afectar la presente investigación. El tiempo con los participantes durante la aplicación fue de diez minutos.

\section{Instrumentos}

El Inventario de Bienestar Espiritual (Spirituality Index of Well-Being - SIWB): desarrollado inicialmente por Daaleman, et al. ${ }^{17}$, contando en su versión original con 
buen nivel de fiabilidad tanto en sus subescalas como en la escala total $(\alpha>0,85)$. Comprende 12 ítems que se responden en un sistema tipo Likert que va de 1 (completamente de acuerdo) a 5 puntos (completamente en desacuerdo), sus dos dimensiones hacen referencia a autoeficacia y esquemas de vida. El rango para cada dimensión va de 6 a 30 puntos y para la escala total de 12 a 60 puntos. A mayor puntuación mayor nivel de bienestar espiritualidad tanto en la escala total como en sus dimensiones.

\section{Procedimiento}

Antes de iniciar la fase de campo de la investigación que implicaba la aplicación del cuestionario SIWB se desarrolló la traducción y adaptación de la versión original inglesa del SIWB al contexto sociolingüístico del español de Colombia que se realizó siguiendo el método de la traducción y re traducción, aceptado por la mayoría de los expertos, se centró en garantizar la equivalencia conceptual de los términos utilizados, esto es, en no alterar el significado original de cada ítem ${ }^{18,19}$. Posteriormente se llevó a cabo la evaluación cultural del instrumento: el trabajo de adaptación cultural consistió en la aplicación del cuestionario SIBW un grupo de diez pacientes crónicos de ambos sexos. Estos luego de responder los cuestionarios fueron entrevistados para identificar palabras o preguntas de difícil comprensión, evaluar la aceptabilidad y hacer comentarios sobre estos de una forma general, registrándose además el tiempo necesario para completarlo y la comprensión respectiva en el sistema de respuesta de cada ítem de los cuestionarios. No se presentaron dificultades que ameritaran cambios, los pacientes reportaron entendimiento y comprensión de los ítems en todos los instrumentos psicométricos evaluados.

Finalmente, con los participantes que acogieron la invitación, se reunieron en las instalaciones de la clínica privada donde fueron encuestados en un consultorio por el psicólogo-investigador según las siguientes fases: 1) se dio lectura al grupo por parte del investigador de la carta de consentimiento informado y se firmó el mismo por cada uno de ellos y 2) se procedió a la aplicación del cuestionario.

\section{Análisis estadísticos}

Los análisis estadísticos se realizaron a través del software estadístico SPSS versión 22. Para esta investigación se realizaron análisis descriptivos de la muestra en sus diferentes variables sociodemográficas y un análisis de adecuación y suficiencia muestral con las pruebas de Kaiser-Meyer-Olkin (esperando puntuaciones superiores a 0,7) y la prueba de esfericidad de Bartlett (esperando una significancia inferior al 0,05 . Sobre la escala en lo que respecta a validez de constructo, se realizó un análisis factorial por medio de la extracción de componentes principales, con rotación Varimax, teniendo en cuenta que la carga inicial de cada reactivo fuera de uno y que la carga factorial superara el 0,5, autovalores superiores a uno, congruencia teórica con los reactivos que lo componen y ajuste a dos factores, mayor carga factorial por reactivo para determinar a qué componente pertenece. Finalmente en lo que respecta a confiabilidad y consistencia, se revisaron las correlaciones inter-elemento esperando correlaciones positivas y superiores a 0,3 , correlaciones elementototal superiores a 0,5 , índice de consistencia con el Alfa de Cronbach, análisis de confiabilidad por mitades con la correlación entre formas y el coeficiente dos mitades de Guttman, y finalmente la revisión de afectaciones negativas al Alfa de Cronbach si se elimina el elemento.

\section{RESULTADOS}

En la Tabla 1 se presentan los datos descriptivos de las variables sociodemográficas y clínicas de la investigación. Se aprecia en este estudio predominio del sexo masculino respecto al femenino, la edad media fue de seis años con un rango entre cuatro a siete años. De acuerdo al estado civil hubo predominio de la muestra en las categorías casado, seguido de viudo. La mayoría alcanzaron un nivel educativo de básica secundaria (bachillerato) y universitario y pertenecen a un estrato socioeconómico colombiano medio alto (nivel cuatro, cinco y seis). De igual modo gran parte de la muestra se encuentran retirados de su vida laboral y un porcentaje menor están dedicados a actividades del hogar. Además gran proposición de los participantes viven con familiares siendo muy pocos quienes viven solos y con amigos. Respecto a las variables clínicas se observa que los pacientes tienen un tiempo de diagnóstico de EPOC de seis años en un rango que oscila entre uno a 24 años de diagnóstico. $\mathrm{La}$ mayoría cuenta con un sistema de salud de medicina pre-pagada, no consumen medicación psiquiátrica pero sí cuentan con un tratamiento medicamentoso específico para su enfermedad. 
Validez de constructo y confiabilidad de la versión en español del Spirituality Index of Well-Being (SIWB) en población colombiana con enfermedad pulmonar crónica

Tabla 1. Descripción de la muestra según las características sociodemográficas

\begin{tabular}{|c|c|c|c|c|c|}
\hline & $n$ & $\%$ & & $n$ & $\%$ \\
\hline Sexo & & & Edad & & \\
\hline Hombre & 42 & 59,2 & Media (D.T) & 72,5 & $(8,5)$ \\
\hline Mujer & 29 & 40,8 & Mín.-Máx. & 49 & 87 \\
\hline Estado civil & & & Nivel educativo & & \\
\hline Soltero & 2 & 2,8 & Primaria & 11 & 15,5 \\
\hline Casado & 46 & 64,8 & Secundaria & 23 & 32,4 \\
\hline $\begin{array}{l}\text { Separado/ } \\
\text { Divorciado }\end{array}$ & 5 & 7 & Tecnología & 10 & 14,1 \\
\hline Unión libre & 3 & 4,2 & Universitario & 23 & 32,4 \\
\hline Viudo & 15 & 21,1 & Posgrado & 4 & 5,6 \\
\hline $\begin{array}{l}\text { Estrato } \\
\text { socioeconómico }\end{array}$ & & & Ocupación & & \\
\hline 2 & 3 & 4,2 & Empleado & 2 & 2,8 \\
\hline 3 & 13 & 18,3 & $\begin{array}{c}\text { Trabajador } \\
\text { independiente }\end{array}$ & 17 & 23,9 \\
\hline 4 & 19 & 26,8 & $\begin{array}{c}\text { Jubilado/ } \\
\text { pensionado }\end{array}$ & 37 & 52,1 \\
\hline 5 & 23 & 32,4 & Hogar & 15 & 21,1 \\
\hline 6 & 13 & 18,3 & & & \\
\hline \multicolumn{6}{|l|}{ Con quien vive } \\
\hline Solo & 6 & 8,5 & & & \\
\hline Con familia & 63 & 88,7 & & & \\
\hline Con amigos & 2 & 2,8 & & & \\
\hline
\end{tabular}

Factores

Clínicos

\begin{tabular}{|c|c|c|c|c|c|}
\hline $\begin{array}{l}\text { Tiempo de } \\
\text { Diagnóstico }\end{array}$ & & & $\begin{array}{l}\text { Sistema de } \\
\text { Salud }\end{array}$ & & \\
\hline Media (D.T) & 70,3 & $(60,7)$ & $\begin{array}{l}\text { Medicina } \\
\text { prepagada }\end{array}$ & 69 & 97,2 \\
\hline Mín.-Máx. & 12 & 288 & $\begin{array}{l}\text { Particular/ } \\
\text { Privada }\end{array}$ & 2 & 2,8 \\
\hline $\begin{array}{l}\text { Toma } \\
\text { medicamentos } \\
\text { psiquiátricos }\end{array}$ & & & $\begin{array}{c}\text { Recibe } \\
\text { tratamiento } \\
\text { específico para } \\
\text { la enfermedad }\end{array}$ & & \\
\hline SI & 6 & 8,5 & SI & 71 & 100 \\
\hline NO & 65 & 91,5 & NO & 0 & 0 \\
\hline
\end{tabular}

Nota: $n=$ número de participantes; D.T= Desviación Típica; Mín. $=$ Mínimo; Máx $=$ Máximo

Tras la aplicación y con el fin de determinar si el tamaño muestral es suficiente para realizar el análisis factorial, se revisan los coeficientes de adecuación muestral de Kaiser-Meyer-Olkin y la Prueba de esfericidad de Bartlett. Los resultados se muestran en la Tabla 2.
Tabla 2. Pruebas de adecuación y suficiencia muestral.

Medida de adecuación muestral de Kaiser-MeyerOlkin.

0,884

Prueba de esfericidad de Bartlett

Chi-cuadrado aproximado 927,869

$$
\begin{array}{cc}
\text { gl } & 66 \\
\text { Sig. } & 0,000
\end{array}
$$

Con los datos anteriores se observa un tamaño de la muestra suficiente para realizar el análisis factorial $(\mathrm{KMO}=0,884$; Bartlett sig $=0,000)$. A continuación se muestra la carga factorial de cada reactivo (Tabla 3 ).

Tabla 3. Carga factorial de cada reactivo

\begin{tabular}{ccc}
\hline Ítem & Inicial & Extracción \\
\hline Item1 & 1,000 & 0,871 \\
Item2 & 1,000 & 0,828 \\
Item3 & 1,000 & 0,870 \\
Item4 & 1,000 & 0,808 \\
Item5 & 1,000 & 0,693 \\
Item6 & 1,000 & 0,613 \\
Item7 & 1,000 & 0,684 \\
Item8 & 1,000 & 0,652 \\
Item9 & 1,000 & 0,752 \\
Item10 & 1,000 & 0,798 \\
Item11 & 1,000 & 0,831 \\
Item12 & 1,000 & 0,773 \\
\hline
\end{tabular}

La extracción hallada (tabla 3) muestra que la totalidad de los reactivos generan la suficiente carga para conservarlos. Dados estos datos, se procedió a realizar el análisis factorial confirmatorio a través de la extracción de componentes principales con rotación Varimax, ajustado a dos componentes como en la versión de Daaleman, et al. ${ }^{17}$. La Tabla 4 muestra los factores obtenidos y la varianza total explicada.

Tabla 4. Varianza total explicada por los factores obtenidos

\begin{tabular}{cccc}
\hline \multirow{2}{*}{ Componente } & \multicolumn{3}{c}{$\begin{array}{c}\text { Suma de las saturaciones al cuadrado de } \\
\text { la rotación }\end{array}$} \\
\cline { 2 - 4 } & Total & \% de la varianza & \% acumulado \\
\hline 1 & 4,666 & 38,884 & 38,884 \\
2 & 4,507 & 37,557 & 76,440 \\
\hline
\end{tabular}

La tabla anterior denota que los dos factores obtenidos explican el $76,440 \%$ de la varianza total acumulada, por lo que se muestra a continuación (Tabla 5) la distribución de los reactivos para cada componente. 
Tabla 5. Matriz de cargas vectoriales.

\begin{tabular}{lcc}
\hline & \multicolumn{2}{c}{ Componente } \\
\cline { 2 - 3 } & $\mathbf{1}$ & $\mathbf{2}$ \\
\hline Item1 & 0,876 & \\
Item2 & 0,901 & \\
Item3 & 0,887 & \\
Item4 & 0,879 & \\
Item5 & 0,702 & \\
Item6 & 0,609 & \\
Item7 & & 0,691 \\
Item8 & & 0,645 \\
Item9 & & 0,851 \\
Item10 & & 0,870 \\
Item11 & & 0,896 \\
Item12 & & 0,805 \\
\hline
\end{tabular}

Nota a: Método de extracción: Análisis de componentes principales.

Nota b: Método de rotación: Normalización Varimax con Kaiser.
Los resultados anteriores muestran que los reactivos 1 al 6 pertenecen al primer factor, mientras que el segundo factor se compone por los reactivos 7 al 12. Ahora, con el fin de clarificar gráficamente los reactivos en un plano, se realizó el gráfico de componentes en espacio rotado (Figura 1), donde se divide el cuadrante superiorderecho a la mitad para más claridad. Dichos resultados muestran que los reactivos que componen cada factor se agrupan claramente, siendo los ítems del 1-6 hacia la parte derecha-abajo del cuadrante, y los reactivos 7-12 al apartado izquierdo-superior del mismo.

En lo que implica a confiabilidad, se halló un alfa de Cronbach general de 0,943, un alfa de 0,936 para el primer componente (ítems del 1 al 6) y de 0,925 para el segundo factor. Adicional, se realizó el análisis de confiabilidad por mitades, obteniendo una correlación entre formas de 0,657 , y un coeficiente dos mitades de Guttman de 0,793.

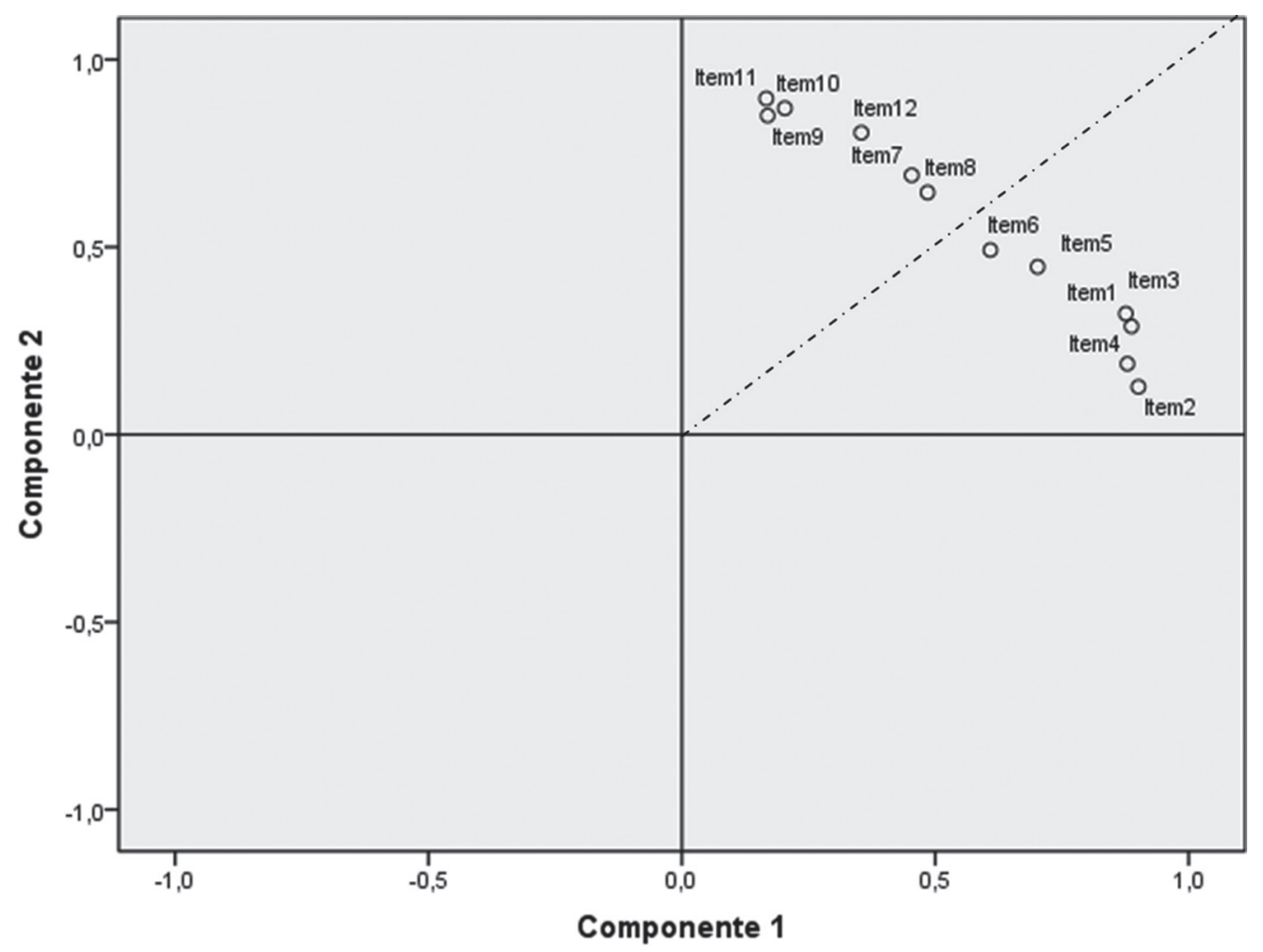

Figura 1. Gráfico de componentes en espacio rotado.

En la Tabla 6 se muestra la matriz de correlaciones inter-elementos con el fin de observar la consistencia entre los reactivos de la escala, mientras que en la Tabla 7 se encuentran las correlaciones elementototal corregida y los alfas si se elimina el elemento. Dichos resultados muestran que existen correlaciones positivas y superiores a 0,3 entre todos los reactivos, altas correlaciones entre los elementos-total y que los alfas son afectados negativamente si se elimina supuestamente cada elemento, lo que reafirma los hallazgos de consistencia de la escala. 
Validez de constructo y confiabilidad de la versión en español del Spirituality Index of Well-Being (SIWB) en población colombiana con enfermedad pulmonar crónica

Tabla 6. Matriz de correlaciones inter-elementos

\begin{tabular}{|c|c|c|c|c|c|c|c|c|c|c|c|c|}
\hline & Item 1 & Item2 & Item3 & Item 4 & Item5 & Item6 & Item 7 & Item 8 & Item 9 & Item 10 & Item 11 & Item 12 \\
\hline Item 1 & --- & 0,826 & 0,908 & 0,822 & 0,703 & 0,558 & 0,572 & 0,618 & 0,459 & 0,515 & 0,453 & 0,553 \\
\hline Item 2 & & --- & 0,866 & 0,780 & 0,592 & 0,540 & 0,395 & 0,459 & 0,271 & 0,346 & 0,354 & 0,460 \\
\hline Item 3 & & & --- & 0,838 & 0,711 & 0,555 & 0,476 & 0,524 & 0,476 & 0,530 & 0,463 & 0,507 \\
\hline Item 4 & & & & --- & 0,651 & 0,528 & 0,473 & 0,469 & 0,351 & 0,399 & 0,362 & 0,470 \\
\hline Item5 & & & & & --- & 0,736 & 0,667 & 0,567 & 0,529 & 0,503 & 0,465 & 0,523 \\
\hline Item6 & & & & & & --- & 0,816 & 0,740 & 0,413 & 0,367 & 0,426 & 0,629 \\
\hline Item 7 & & & & & & & --- & 0,833 & 0,560 & 0,538 & 0,549 & 0,703 \\
\hline Item 8 & & & & & & & & --- & 0,503 & 0,516 & 0,528 & 0,728 \\
\hline Item 9 & & & & & & & & & --- & 0,844 & 0,778 & 0,608 \\
\hline Item 10 & & & & & & & & & & --- & 0,884 & 0,699 \\
\hline Item11 & & & & & & & & & & & --- & 0,806 \\
\hline Item12 & & & & & & & & & & & & --- \\
\hline
\end{tabular}

Tabla 7. Estadísticos total-elemento

\begin{tabular}{lcc}
\hline & $\begin{array}{c}\text { Correlación elemento- } \\
\text { total corregida }\end{array}$ & $\begin{array}{c}\text { Alfa de Cronbach si se } \\
\text { elimina el elemento }\end{array}$ \\
\hline Item1 & 0,815 & 0,935 \\
Item2 & 0,677 & 0,940 \\
Item3 & 0,800 & 0,936 \\
Item4 & 0,709 & 0,939 \\
Item5 & 0,769 & 0,937 \\
Item6 & 0,729 & 0,938 \\
Item7 & 0,761 & 0,937 \\
Item8 & 0,751 & 0,938 \\
Item9 & 0,665 & 0,941 \\
Item10 & 0,707 & 0,939 \\
Item11 & 0,699 & 0,939 \\
Item12 & 0,775 & 0,937 \\
\hline
\end{tabular}

\section{DISCUSIÓN}

Actualmente el modelo Bio-Psico-Social ha sido aceptado como un marco para la comprensión de las necesidades de los enfermos crónicos más que el tradicional enfoque bio-medico; dentro este modelo se considera la enfermedad en el contexto de una compleja interaccion de factores biologicos, psicológicos, sociales y espirituales ${ }^{19}$.
Las posturas actuales sobre salud que plantea la Organización Mundial de la Salud, entendida como un estado de total bienestar en el individuo ${ }^{20}$, la calidad de vida relacionada con la salud se ha transformado en un indicador importante de la salud subjetiva, que permite encarar procedimientos y procesos de intervención previa a enfermedades, o la evaluación percibida ante una patología crónica.

Por otro lado la importancia de la espiritualidad en la vida de los pacientes crónicos no puede ser pasada por alto ya que este factor puede servir como componente clave para construir el marco por el cual los pacientes guían la toma de decisiones durante su padecimiento ${ }^{13}$.

En ese sentido, al analizar los resultados obtenidos se halló que la escala SIWB muestra una estructura bifactorial: una sub escala de autoeficacia (ítems 1-6) y una sub escala de esquemas vitales (ítems 7-12). De igual forma se hallan alfas altos, correlaciones positivas entre todos los reactivos y afectaciones ante hipotéticas eliminaciones de reactivos, dando cuenta de su consistencia.

Dichos factores hallados y sus datos de confiabilidad concuerdan por lo tanto con los factores inicialmente encontrados en los hallazgos de Daaleman, et al. ${ }^{17}$ tanto en factores como en reactivos y datos de confiabilidad, en población de habla inglesa en los Estados Unidos, lo que habla de la continuidad de los factores 
independientes al contexto cultural, siendo en este caso el colombiano.

Precisamente y frente a las implicaciones para la aplicación de la escala en sujetos con EPOC, la escala analizada en esta investigación permite darle al profesional de la salud una evaluación sobre la espiritualidad de dichos pacientes, siendo una evaluación libre de la religiosidad o la postura sobre un Dios en específico ${ }^{17}$, lo que reafirma su importante utilidad y su aplicabilidad en contextos clínicos y con diferentes tipos de pacientes, dando luces sobre un componente clave para construir el marco decisional durante su enfermedad ${ }^{13}$.

Ahora, en lo que implica a la validez externa de la presente investigación, se homogeneizó el protocolo de aplicación a todos los participantes y se aplicó el cuestionario con base en los lineamientos originales de los autores ${ }^{17}$, y dado que se controlaron variables en los participantes que pudiesen alterar los resultados (criterio de la enfermedad, homogeneidad de proveniencia, trastornos psiquiátricos, entre otros), los datos pueden ser generalizables a otros pacientes con EPOC en Colombia, sin embargo, algunas limitaciones de este estudio radican en que la muestra fue obtenida de una sola ciudad, por lo que se recomienda replicar este estudio con muestras de otras ciudades para verificar los hallazgos de este estudio en pro de la generalización de sus resultados. Esto se plantea porque, si bien se considera que existe una invarianza factorial de la escala, más cuando los resultados fueron estadísticamente robustos, y que son similares a los estudios originales de la escala, también debe tenerse en cuenta que hay factores que pueden afectar la validez del constructo (étnico-culturales, diferenciación en las regiones colombianas, respuesta diferencial de cada reactivo, etc).

De igual forma el presente es un análisis factorial, que arroja importantes resultados, pero es necesario rectificar estos hallazgos por medio de un análisis factorial confirmatorio, análisis que desborda esta investigación. Adicionalmente, si bien se reconoce la dificultad de conseguir muestras clínicas con un número amplio de sujetos, se recomienda realizar este estudio con una muestra mayor, que permita rectificar estos hallazgos, preferiblemente con matrices factoriales más robustas, como puede ser la matriz policórica ${ }^{21,22}$.

Finalmente se concluye de este estudio que la escala SIWB se ajusta a la población colombiana, tanto en términos de validez de constructo como de confiabilidad, lo que favorecería su utilización nacional tanto en investigación en ambientes clínicos y hospitalarios, y posibilidades de estudios transculturales, dados los hallazgos encontrados.

\section{REFERENCIAS}

1. Barnes P. Chronic obstructive pulmonary disease. NEJM. 2000; 343: 269-280. DOI: 10.1056/ NEJM200007273430407.

2. De Calvo E, Martin A. Atención al paciente con asma y EPOC en la consulta del medico de familia. Barcelona: Masson; 2002.

3. Parmet S. Enfermedad pulmonar obstructiva crónica. JAMA. 2003; 290: 17, 2362

4. Organización mundial de la Salud. Preventing chronic diseases: a vital investment: Geneve: 2013.

5. Ministerio de Salud. Enfermedad Pulmonar Obstructiva Crónica-EPOC. Bogotá: 2013.

6. Medinas MM, Más C, Renom F. Estrés en ancianos hospitalizados con enfermedad respiratoria crónica: Valoración en el hospital sociosanitario. Rev Española Geriat Gerontol. 2009; 44(3): 130-136. DOI:10.1016/j.regg.2009.01.006

7. Kelly C, Lynes D. Psychological effects of chronic lung disease. Nurs Times. 2008; 104: 82-85.

8. Wilson I. Depression in patients with COPD. Int J Chron Obstruct Pulmon Dis. 2008; 1(1): 61-64.

9. Maurer J, Rebbapragada V, Borson S, Goldstein R, Kunik ME, Yohannes AM, et al. Anxiety and depression in COPD: current understanding, unanswered questions, and research needs. Chest. 2008; 134(4 Suppl): 43-56. DOI: 10.1378/ chest.08-0342.

10. Pargament KI. The Psychology of Religion and Coping: Theory, Research, Practice. New York, NY: Guilford Press; 1997.

11. Muñoz A. ¿Por qué las creencias religiosas funcionan como recurso de afrontamiento? El modelo de K.I. Pargament. Encuent Psicolog Soc. 2003; 1(2): 280-283.

12. Muñoz A, Moreno M. Relevancia y variaciones en las estrategias de afrontamiento religiosoespirituales de jóvenes y adultos. Encuent Psicolog Soc. 2003; 1(5): 273-277.

13. Koenig HG, McCullough M, Larson DB. Handbook of religion and health: a century of research reviewed. New York: Oxford University Press; 2001.

14. Bredle J, Salsman J, Debb S, Arnold B, Cella D. Spiritual Well-Being as a component of healthrelated quality of life: The functional assessment of chronic illness therapy Well Being Scale (FACIT- 
Sp). Religions. 2011; 2: 77-94. DOI: 10.3390/ REL2010077.

15. Strada EA, Homel P, Tennstedt S, Billings JA, Portenoy RK. Spiritual well-being in patients with advanced heart and lung disease. Palliat Support Care. 2013; 11(3): 205-213. DOI: 10.1017/ S1478951512000065.

16. Quiceno J, Vinaccia S. La salud en el marco de la psicología de la religión y la espiritualidad. Diversitas. 2009; 5(2): 321-336.

17. Daaleman T, Frey B. The spiritual index well-being: A new instrument for health related quality of life. Ann Fam Med. 2008; 2(5): 499-503.

18. Montero I, Leon O. A guide for naming research studies in Psychology. Int J Clin Health Psychol.
2007; 7(3): 847-862.

19. Badía X, Salamero M, Alonso J. La medida de la salud. Barcelona: Edimac; 2002.

20. Richardson P. Spiritually, religion and palliative care. Ann Palliat Med. 2014; 3(3): 150-159. DOI: 10.3978/j.issn.2224-5820.2014.07.05.

21. Bandalos DL, Finney SJ. Factor Analysis: Exploratory and Confirmatory. En Hancock GR, Mueller RO. (Eds.), Reviewer's guide to quantitative methods. Routledge: New York. 2010.

22. Lloret-Segura S, Ferreres-Traver A, HernándezBaeza A, Tomás-Marco I. El análisis factorial exploratorio de los ítems: una guía práctica, revisada y actualizada. Anal Psicol. 2014; 30(3); 1151-1169. 


\section{Apéndice A.}

Inventario de bienestar espiritual SIWB

Daaleman TP, Frey BB (2004).

Cual enunciado describe mejor sus sentimientos y deseos?

\begin{tabular}{|c|c|c|c|c|c|}
\hline & $\begin{array}{l}\text { COMPLETAMENTE } \\
\text { DE ACUERDO }\end{array}$ & DE ACUERDO & $\begin{array}{l}\text { NI ACUERDO NI EN } \\
\text { DESACUERDO }\end{array}$ & EN DESACUERDO & $\begin{array}{l}\text { COMPLETAMENTE } \\
\text { EN DESACUERDO }\end{array}$ \\
\hline & 1 & 2 & 3 & 4 & 5 \\
\hline \multicolumn{6}{|c|}{1 No hay mucho que pueda hacer para ayudarme a mí mismo } \\
\hline & 1 & 2 & 3 & 4 & 5 \\
\hline \multicolumn{6}{|c|}{ Frecuentemente, no hay forma de que yo pueda completar algo que haya iniciado } \\
\hline & 1 & 2 & 3 & 4 & 5 \\
\hline \multicolumn{6}{|c|}{3 No puedo comenzar a entender mis problemas } \\
\hline & 1 & 2 & 3 & 4 & 5 \\
\hline \multirow[t]{2}{*}{4} & \multicolumn{5}{|c|}{ Siento que las cosas me sobrepasan cuando tengo problemas y dificultades personales } \\
\hline & 1 & 2 & 3 & 4 & 5 \\
\hline \multirow[t]{2}{*}{5} & \multicolumn{5}{|c|}{ No sé cómo comenzar a resolver mis problemas } \\
\hline & 1 & 2 & 3 & 4 & 5 \\
\hline \multirow[t]{2}{*}{6} & \multicolumn{5}{|c|}{ No hay mucho que yo pueda hacer para marcar una diferencia en mi vida } \\
\hline & 1 & 2 & 3 & 4 & 5 \\
\hline \multirow[t]{2}{*}{7} & \multicolumn{5}{|c|}{ Todavía no le encuentro un propósito a mi vida } \\
\hline & 1 & 2 & 3 & 4 & 5 \\
\hline \multirow[t]{2}{*}{8} & \multicolumn{5}{|c|}{ No me conozco a mí mismo, de donde vengo o a donde voy } \\
\hline & 1 & 2 & 3 & 4 & 5 \\
\hline \multirow[t]{2}{*}{9} & \multicolumn{5}{|c|}{ Me hace falta un propósito en mi vida } \\
\hline & 1 & 2 & 3 & 4 & 5 \\
\hline \multicolumn{6}{|c|}{10 En este mundo, no sé qué lugar ocupo } \\
\hline & 1 & 2 & 3 & 4 & 5 \\
\hline \multicolumn{6}{|c|}{11 Estoy muy lejos de entender el significado de mi vida } \\
\hline & 1 & 2 & 3 & 4 & 5 \\
\hline \multicolumn{6}{|c|}{12 Hay un gran vacío en mi vida en este momento } \\
\hline & 1 & 2 & 3 & 4 & 5 \\
\hline
\end{tabular}

